\title{
\%
}

Subdirección General de Estudios y

Evaluación de Instrumentos de Política Comercial*

\section{EFICACIA DEL APOYO A LA INTERNACIONALIZACIÓN La percepción de los exportadores}

Conocer la percepción de las empresas sobre los instrumentos de apoyo a la internacionalización es esencial para mejorar su diseño y adaptarlo a sus necesidades específicas. La Encuesta Piloto sobre Apoyo a la Internacionalización, realizada a empresas que exportan regularmente, es un avance en esta dirección. La encuesta aporta una visión global sobre el conocimiento que tienen las empresas exportadoras de los servicios de ICEX y CESCE y la valoración que hacen de ellos. Sus resultados revelan niveles de satisfacción elevados, apuntan a la conveniencia de reforzar la visibilidad de estos instrumentos y validan el objetivo de adaptarlos en mayor medida a las necesidades específicas de los usuarios en línea con las actuaciones que están aplicando tanto ICEX como CESCE.

Palabras clave: comercio exterior, internacionalización, pyme, exportación.

Clasificación JEL: F13, F43, L21, L25.

\section{Introducción}

Para mejorar el diseño de los instrumentos de internacionalización y, en última instancia, potenciar su eficacia, es esencial conocer cómo los perciben las empresas.

En particular, es necesario determinar hasta qué punto los instrumentos que se ofrecen son conocidos por las empresas, en qué grado los utilizan o por qué no lo hacen, y en qué medida los resultados que obtienen responden a sus expectativas.

\footnotetext{
* Secretaría de Estado de Comercio. Versión de abril de 2020.

DOI: https://doi.org/10.32796/bice.2020.3123.7001
}

La eficacia de los distintos instrumentos puede variar en función del sector de actividad, volumen de exportación y otras características de las empresas hacia las que se dirigen. Las percepciones de los distintos tipos de empresa sobre su eficacia pueden ayudar a un diseño más adecuado a sus características particulares.

Ofrecer un apoyo a la internacionalización adaptado al perfil de las empresas es, precisamente, el primer eje de la Estrategia de Internacionalización de la Economía Española 2017-2027, que tiene como objetivo general consolidar la contribución positiva del sector exterior al crecimiento económico e impulsar la creación de empleo (Ministerio de Economía, Industria y Competitividad, 2017; $\triangleright$ 
Subdirección General de Análisis y Estrategia de Internacionalización, 2017).

La citada estrategia, que se desarrolla a través de planes de actuación bienales, incluye, entre sus principios rectores, los de transparencia y evaluación. En aplicación de estos principios prevé la realización de ejercicios de evaluación periódicos que permitan seguir el grado de cumplimiento de las metas fijadas, así como su eficiencia y efectividad. Estos procesos se realizarán cada dos años, en el caso de los planes de acción, y cada cinco años, en el caso de la estrategia.

Para avanzar en el conocimiento de la percepción de las empresas sobre los instrumentos de apoyo a la internacionalización y contribuir a estos ejercicios de transparencia y evaluación es preciso contar con instrumentos que permitan su medición. Con este objetivo, en el cuarto trimestre de 2019, se incluyó en la Encuesta de Coyuntura de la Exportación un módulo sobre la eficacia de los principales instrumentos de apoyo a la internacionalización, denominado Encuesta Piloto sobre Apoyo a la Internacionalización (EPAI).

En este artículo se resumen los principales resultados de esta encuesta piloto. En el epígrafe 2 se realiza una breve descripción de la metodología de la EPAl, enfocada a captar la percepción de las empresas que exportan regularmente sobre dos grandes vehículos de apoyo a la internacionalización: ICEX y CESCE. A continuación, en el epígrafe 3 , se presentan los resultados sobre la cobertura y eficacia de ICEX y sobre las razones por las que, en su caso, las empresas no utilizan sus servicios. En el epígrafe 4 se estudian los resultados de cobertura y eficacia para CESCE. El artículo finaliza con un apartado en el que se resumen las conclusiones alcanzadas.

\section{La Encuesta Piloto sobre Apoyo a la Internacionalización}

La EPAI se apoya en un cuestionario muy sencillo, de siete preguntas (ver Anexo), orientado a investigar cómo perciben las empresas exportadoras regulares los dos vehículos de uso más general para el apoyo a la internacionalización: ICEX y CESCE.

No se pretende una cuantificación precisa de la eficacia o del impacto de las distintas ayudas, programas, servicios o instrumentos que cada una de estas instituciones ofrece a las empresas como apoyo a su proceso de internacionalización. Se trata de obtener una visión general sobre el grado de conocimiento de estas instituciones entre el colectivo de empresas que exportan de forma regular, la utilización que hacen de estos instrumentos, su satisfacción con los resultados obtenidos y, para el caso de ICEX, las razones por las que, en su caso, han decidido no utilizar estos servicios, a pesar de conocerlos.

Para la realización de esta experiencia piloto se ha incluido un módulo adicional en la Encuesta de Coyuntura de la Exportación, que trimestralmente realiza la Secretaría de Estado de Comercio del Ministerio de Industria Comercio y Turismo. Los principales datos técnicos de esta encuesta se resumen en el Recuadro 1. El trabajo de campo se ejecutó en el cuarto trimestre de 2019.

Un dato a destacar es que la encuesta cubre un universo de empresas cuyas exportaciones, en el periodo 2014-2017, supusieron el $96,1 \%$ de las exportaciones de bienes españolas. Puede considerarse, por tanto, altamente representativa del volumen total de exportación de bienes.

Sin embargo, a efectos del conocimiento de las diferentes necesidades de las empresas internacionalizadas o con potencial para la $D$ 
internacionalización, debe tenerse presente que la EPAI no está exenta de limitaciones. Entre ellas, no incluye a las empresas que exportan servicios, a las que exportan de forma no regular o por debajo del umbral establecido para obtener la muestra, a las que acaban de iniciarse en la exportación o aún no lo han hecho, pese a tener potencial para ello, o a las que se internacionalizan, principalmente, a través de la inversión en el exterior.

\section{Recuadro 1 \\ Metodología de la EPAI}

\section{Ámbito}

- Poblacional: empresas españolas que realizan de forma continua operaciones de exportación de mercancías.

- Temporal: se lleva a cabo con carácter trimestral; el periodo de referencia de la información habitual es el trimestre en curso, pero para el módulo piloto se solicitó información desde inicios de 2017 hasta la realización de la encuesta.

- Geográfico: el territorio del Estado español.

Unidades estadísticas: las empresas que realizan de manera continua actividades de exportación de bienes, que son asimismo las unidades informantes.

\section{Marco y directorio}

- Se considera que una empresa tiene una actividad exportadora continua si realizó operaciones de exportación por valor superior a los 30.000 euros anuales en cada uno de los años del periodo 2014-2017. Para determinar qué empresas cumplen esta condición, se utiliza la información de comercio exterior de mercancías (datos de comercio declarado) del Departamento de Aduanas e Impuestos Especiales de la Agencia Estatal de la Administración Tributaria. A partir de dicha información se actualiza el directorio que sirve de base para obtener la muestra trimestral.

- El directorio consta de 25.765 empresas, que son las responsables del 96,1\% de las exportaciones españolas de mercancías para el periodo 2014-2017.

\section{Diseño muestral}

- Tamaño muestral: la muestra se compone de un total de 1.900 unidades (empresas).

- Tipo de muestreo: muestreo aleatorio estratificado.

- Estratificación: se utilizan dos variables para estratificar la población de empresas exportadoras incluidas en el directorio, sector y tamaño. Las empresas se asignan a los diferentes sectores en función del tipo de bienes que exportan (producto principal), considerando nueve sectores. La variable de tamaño utilizada es el valor anual medio de las exportaciones de las empresas en el periodo 2014-2017. Se consideran un total de cuatro categorías o tamaños. 


\section{Resultados para las ayudas, programas y servicios de ICEX}

Los resultados obtenidos (Cuadro 1) revelan que el $18,5 \%$ de las empresas españolas que exportan regularmente ${ }^{1}$ valora positivamente la incidencia que han tenido los servicios y programas de apoyo a la internacionalización ofrecidos por ICEX (en adelante, servicios de ICEX), desde 2017 hasta finales de 2019, en su proceso de internacionalización. Este resultado se obtiene calculando el porcentaje que representan las empresas que conocen, han

1 La definición de empresas que exportan regularmente a efectos de la EPAl es diferente de la empleada en otras publicaciones de la Secretaría de Estado de Comercio, como el Informe Mensual de Comercio Exterior. En el caso de la EPAl, como se expone en el Recuadro 1, se trata de empresas cuya la regularidad viene definida por el comportamiento en el periodo 2014-2017 y se exige la superación anual de un umbral de exportaciones declaradas. En el caso del Informe Mensual de Comercio Exterior, se trata de operadores (no necesariamente empresas) cuya regularidad se refiere a haber declarado exportaciones en el periodo de referencia y en los tres años anteriores. No se exige la superación de un umbral. utilizado y han quedado satisfechas con los servicios de ICEX sobre el total de empresas que forman parte del ámbito de investigación.

Por sectores, aquellos en los que hay un mayor porcentaje de empresas regulares que se han beneficiado desde 2017 a 2019 de los servicios de ICEX son los de alimentación, bebidas y tabaco y bienes de consumo duradero (31,5\% y $26,9 \%$, respectivamente). Aquellos sectores en los que hay un menor porcentaje de empresas que se han beneficiado de ICEX son los de productos energéticos y materias primas $(0,5 \%$ y $3,8 \%$, respectivamente).

Por intervalos de valor de exportación, destaca, con un $25,2 \%$, el porcentaje de empresas que perciben un resultado positivo de ICEX en el intervalo medio-alto (de 3 a 15 millones de euros).

El grado de satisfacción de las empresas regulares que declaran conocer y haber utilizado los servicios de ICEX es muy elevado en $D$

CUADRO 1

PERCEPCIÓN DE LAS EMPRESAS EXPORTADORAS SOBRE LA COBERTURA Y EFICACIA DE ICEX

\begin{tabular}{|c|c|c|c|c|}
\hline \multicolumn{5}{|c|}{ Porcentaje de empresas del sector } \\
\hline Sectores & Conocimiento & Utilización & Satisfacción & +/Total \\
\hline Alimentación, bebidas y tabaco & 80,7 & 42,2 & 92,7 & 31,5 \\
\hline Productos energéticos & 47,4 & 1,0 & 94,3 & 0,5 \\
\hline Materias primas & 65,3 & 5,8 & 100,0 & 3,8 \\
\hline Semimanufacturas no químicas & 79,3 & 24,9 & 97,4 & 19,2 \\
\hline Productos químicos & 72,0 & 21,1 & 100,0 & 15,2 \\
\hline Bienes de equipo & 71,5 & 32,7 & 95,6 & 22,3 \\
\hline Sector del automóvil & 49,7 & 21,3 & 100,0 & 10,6 \\
\hline Bienes de consumo duradero & 87,5 & 30,8 & 100,0 & 26,9 \\
\hline Manufacturas de consumo & 69,4 & 35,1 & 94,5 & 23,0 \\
\hline Total & 67,9 & 28,4 & 95,9 & 18,5 \\
\hline \multicolumn{5}{|c|}{ Porcentaje de empresas del intervalo de exportación } \\
\hline Millones de $€$ & Conocimiento & Utilización & Satisfacción & $+/$ Total \\
\hline $0,03-0,6$ & 61,5 & 27,6 & 95,6 & 16,2 \\
\hline $0,6-3$ & 68,2 & 31,0 & 93,3 & 19,7 \\
\hline 3 a 15 & 76,0 & 33,6 & 98,9 & 25,2 \\
\hline Más de 15 & 66,5 & 27,1 & 95,3 & 17,2 \\
\hline Total & 67,9 & 28,4 & 95,9 & 18,5 \\
\hline
\end{tabular}

Fuente: EPAI. Secretaría de Estado de Comercio. Ministerio de Industria Comercio y Turismo. 
todos los sectores e intervalos de exportación. Globalmente, el $95,9 \%$ de los usuarios dicen estar satisfechos con el resultado. Por sectores, es especialmente destacable el grado de satisfacción de los usuarios de ICEX en los de materias primas, productos químicos, automóvil y bienes de consumo duradero, donde el porcentaje de satisfacción es pleno (100\%). Por intervalos de exportación, los usuarios con mayor grado de satisfacción $(98,9 \%)$ se sitúan en el de 3 a 15 millones de euros.

En cuanto a la visibilidad de ICEX, un 67,9\% de las empresas exportadoras regulares declaran conocer los servicios de ICEX. Sectorialmente, los porcentajes de visibilidad más elevados se dan en los sectores de bienes de consumo duradero $(87,5 \%)$ y alimentación, bebidas y tabaco $(80,7 \%)$; y los más reducidos, en productos energéticos $(47,4 \%)$ y sector del automóvil $(49,7 \%)$, dos sectores que, por su estructura, seguramente necesiten menos apoyo público para acceder a los mercados exteriores. Por intervalos de exportación, destaca el conocimiento de ICEX entre las empresas que exportan volúmenes anuales entre 3 y 15 millones de euros (76\%).

El porcentaje de empresas regulares que conociendo ICEX utilizan sus servicios es del 28,4 . Al valorar este dato debe tenerse presente que la encuesta se realiza a exportadores regulares que, por lo tanto, es probable que tengan una presencia ya consolidada en sus mercados de exportación, con clientes y canales de distribución ya establecidos, y no precisen recurrir a nuevos servicios de apoyo para mantenerse en ellos. Este porcentaje es muy variable entre sectores, con un máximo del $42,2 \%$ entre las empresas de alimentación, bebidas y tabaco y un mínimo del 1,0\% en productos energéticos. Por intervalos de exportación, al igual que en el caso del conocimiento y la satisfacción, el grado de uso es más elevado $(33,6 \%)$ entre las empresas regulares que exportan de 3 a 15 millones de euros al año.

Los resultados anteriores apuntan a la necesidad de profundizar en las razones por las que las empresas que conocen ICEX no han utilizado sus servicios (Cuadro 2). Como ya se ha señalado, el hecho de que la encuesta se realice entre exportadores regulares puede determinar una menor necesidad de recurrir a los servicios de apoyo que ofrece ICEX, frente a empresas que no exportan regularmente. Los resultados de la EPAI parecen confirmar esta idea. Así, la mayor parte de las empresas indica que no necesita dichos servicios $(64,8 \%)$, mientras que el $22,7 \%$ manifiesta que los servicios ofrecidos no se adaptan a sus necesidades. Otras razones, como que otras entidades públicas o privadas ofrezcan un apoyo mejor $(7,7 \%)$, la existencia de requerimientos excesivos $(2,7 \%)$ o el coste $(2,2 \%)$, tienen menor importancia, de acuerdo con las opiniones manifestadas por las empresas. El bajo porcentaje de empresas que dan estas respuestas sugiere que los servicios de apoyo que oferta ICEX están entre los mejores disponibles, que se puede acceder a ellos de forma eficiente y que presentan una elevada relación calidad-coste ${ }^{2}$.

Por sectores, destaca el porcentaje de empresas que afirman no necesitar los servicios de ICEX en automóvil $(78,0 \%)$ y productos energéticos $(74,9 \%)$, lo que, como se ha señalado, puede responder a las características estructurales propias de estos sectores.

\footnotetext{
2 A este respecto cabe también señalar que, en muchos casos, ICEX interviene directa o indirectamente en la cadena de valor de la prestación de servicios por parte de otros actores, aun cuando pierda total o parcialmente la visibilidad ante la empresa: por ejemplo, cuando la comunidad autónoma actúa de agrupador ante las empresas de su demarcación en pabellones organizados por ICEX en eventos feriales, programas de ayuda incluidos en los planes sectoriales con las asociaciones, rol de la cámara de comercio en la agrupación de empresas en misiones canalizadas como servicios personalizados prestados por la red de Oficinas Económicas y Comerciales, etcétera.
} 
Subdirección General de Estudios y Evaluación de Instrumentos de Política Comercial

CUADRO 2

RAZONES POR LAS QUE LAS EMPRESAS QUE CONOCEN ICEX NO LO UTILIZAN

\begin{tabular}{|c|c|c|c|c|c|}
\hline \multicolumn{6}{|c|}{ Porcentaje de empresas del sector } \\
\hline Sectores & No lo necesitan & $\begin{array}{l}\text { Otros lo hacen } \\
\text { mejor }\end{array}$ & $\begin{array}{l}\text { Instrumentos } \\
\text { no adaptados }\end{array}$ & $\begin{array}{l}\text { Requerimientos } \\
\text { excesivos }\end{array}$ & Muy caro \\
\hline Alimentación, bebidas y tabaco & 56,8 & 6,7 & 32,2 & 2,0 & 2,4 \\
\hline Productos energéticos & 74,9 & 0,0 & 24,8 & 0,2 & 0,0 \\
\hline Materias primas & 60,5 & 7,2 & 29,4 & 2,6 & 0,3 \\
\hline Semimanufacturas no químicas & 57,7 & 6,6 & 28,1 & 3,3 & 4,3 \\
\hline Productos químicos & 67,7 & 4,3 & 23,7 & 1,4 & 2,8 \\
\hline Bienes de equipo & 59,1 & 10,0 & 21,9 & 5,6 & 3,4 \\
\hline Sector del automóvil & 78,0 & 15,0 & 6,0 & 1,1 & 0,0 \\
\hline Bienes de consumo duradero & 58,2 & 9,5 & 32,0 & 0,3 & 0,0 \\
\hline Manufacturas de consumo & 67,6 & 5,2 & 21,9 & 4,0 & 1,2 \\
\hline Total & 64,8 & 7,7 & 22,7 & 2,7 & 2,2 \\
\hline \multicolumn{6}{|c|}{ Porcentaje de empresas del intervalo de exportación } \\
\hline Millones de $€$ & No lo necesitan & $\begin{array}{l}\text { Otros lo hacen } \\
\text { mejor }\end{array}$ & $\begin{array}{l}\text { Instrumentos } \\
\text { no adaptados }\end{array}$ & $\begin{array}{l}\text { Requerimientos } \\
\text { excesivos }\end{array}$ & Muy caro \\
\hline $0,03-0,6$ & 55,4 & 2,3 & 26,3 & 8,3 & 7,7 \\
\hline $0,6-3$ & 57,7 & 6,4 & 25,2 & 6,8 & 3,9 \\
\hline 3 a 15 & 56,0 & 7,2 & 27,9 & 6,7 & 2,2 \\
\hline Más de 15 & 67,1 & 7,9 & 21,4 & 1,5 & 2,0 \\
\hline Total & 64,8 & 7,7 & 22,7 & 2,7 & 2,2 \\
\hline
\end{tabular}

En lo que se refiere a los intervalos de exportación es destacable que los factores de requerimientos y coste de acceso a los servicios, aun manteniéndose en niveles reducidos, son percibidos como razones para no utilizar los servicios de ICEX por porcentajes más elevados de las empresas situadas en los tramos más bajos de exportación (de 30.000 a 600.000 euros y de 600.000 a 3 millones de euros).

Las diferencias sectoriales y por intervalos de exportación detectadas sugieren que la cobertura y eficacia de los servicios de apoyo a la internacionalización ofertados por ICEX está vinculada a características propias de las empresas, relacionadas con su capacidad exportadora y con el sector en que actúan. Los Cuadros 3 y 4 ofrecen una visión más desagregada, cruzando las dimensiones de sector y de intervalo de exportación.
En términos generales, se observa que el conocimiento de los servicios de ICEX es más frecuente en los intervalos de exportación elevados (de 3 a 15 y de más de 15 millones de euros) en prácticamente todos los sectores y que, en la mayor parte, alcanza el máximo en el intervalo de 3 a 15 millones de euros. El porcentaje de utilización es también, para algunos sectores, relativamente el más elevado en este intervalo (así ocurre en alimentación, bebidas y tabaco; productos energéticos; semimanufacturas no químicas, y bienes de equipo). Sin embargo, en otros sectores es mayor el porcentaje de empresas que utilizan los servicios de ICEX en los intervalos menores de exportación (materias primas, productos químicos, automóvil, bienes de consumo duradero y manufacturas de consumo). Los índices de satisfacción son muy elevados (próximos o $D$ 
CUADRO 3

COBERTURA Y EFICACIA DE ICEX. DESGLOSE POR SECTOR E INTERVALOS DE EXPORTACIÓN

Porcentaje de empresas del sector e intervalo de exportación (millones de $€$ )

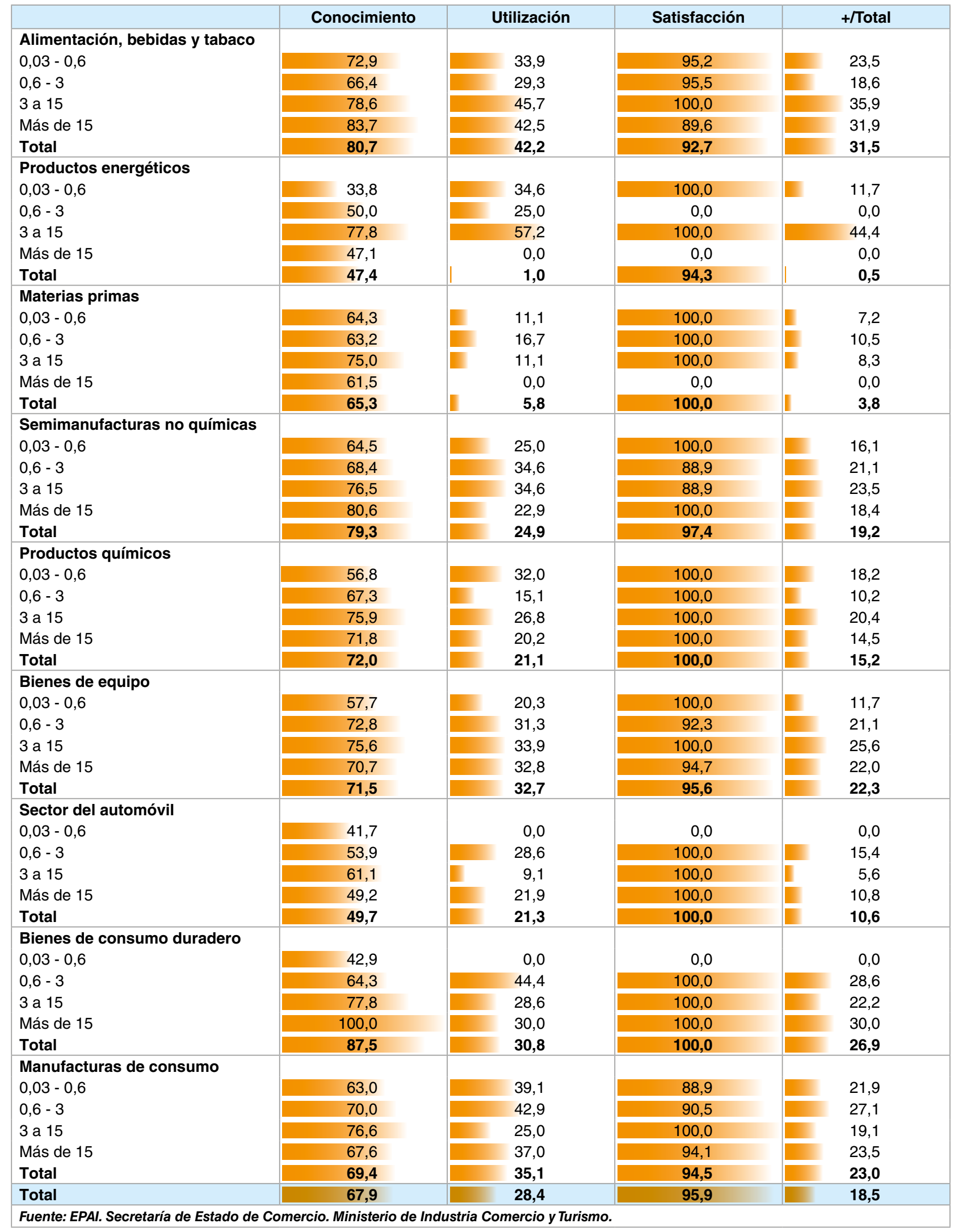


Subdirección General de Estudios y Evaluación de Instrumentos de Política Comercial

CUADRO 4

RAZONES POR LAS QUE LAS EMPRESAS QUE CONOCEN ICEX NO LO UTILIZAN. DESGLOSE POR SECTOR E INTERVALOS DE EXPORTACIÓN Porcentaje de empresas del sector e intervalo de exportación (millones de $€$ )

\begin{tabular}{|c|c|c|c|c|c|}
\hline & No lo necesitan & $\begin{array}{l}\text { Otros lo hacen } \\
\text { mejor }\end{array}$ & $\begin{array}{l}\text { Instrumentos } \\
\text { no adaptados }\end{array}$ & $\begin{array}{l}\text { Requerimientos } \\
\text { excesivos }\end{array}$ & Muy caro \\
\hline \multicolumn{6}{|c|}{ Alimentación, bebidas y tabaco } \\
\hline $0,03-0,6$ & 48,8 & 7,3 & 24,4 & 7,3 & 12,2 \\
\hline $0,6-3$ & 50,9 & 9,4 & 34,0 & 3,8 & 1,9 \\
\hline 3 a 15 & 59,1 & 15,9 & 22,7 & 2,3 & 0,0 \\
\hline Más de 15 & 56,9 & 3,1 & 35,4 & 1,5 & 3,1 \\
\hline Total & 56,8 & 6,7 & 32,2 & 2,0 & 2,4 \\
\hline \multicolumn{6}{|c|}{ Productos energéticos } \\
\hline $0,03-0,6$ & 50,0 & 0,0 & 0,0 & 0,0 & 50,0 \\
\hline $0,6-3$ & 66,7 & 0,0 & 33,3 & 0,0 & 0,0 \\
\hline 3 a 15 & 66,7 & 0,0 & 0,0 & 33,3 & 0,0 \\
\hline Más de 15 & 75,0 & 0,0 & 25,0 & 0,0 & 0,0 \\
\hline Total & 74,9 & 0,0 & 24,8 & 0,2 & 0,0 \\
\hline \multicolumn{6}{|c|}{ Materias primas } \\
\hline $0,03-0,6$ & 37,5 & 0,0 & 37,5 & 12,5 & 12,5 \\
\hline $0,6-3$ & 50,0 & 0,0 & 30,0 & 20,0 & 0,0 \\
\hline 3 a 15 & 62,5 & 0,0 & 37,5 & 0,0 & 0,0 \\
\hline Más de 15 & 62,5 & 12,5 & 25,0 & 0,0 & 0,0 \\
\hline Total & 60,5 & 7,2 & 29,4 & 2,6 & 0,3 \\
\hline \multicolumn{6}{|c|}{ Semimanufacturas no químicas } \\
\hline $0,03-0,6$ & 53,3 & 0,0 & 26,7 & 6,7 & 13,3 \\
\hline $0,6-3$ & 52,9 & 17,7 & 23,5 & 0,0 & 5,9 \\
\hline 3 a 15 & 47,1 & 5,9 & 41,2 & 5,9 & 0,0 \\
\hline Más de 15 & 59,4 & 6,3 & 26,6 & 3,1 & 4,7 \\
\hline Total & 57,7 & 6,6 & 28,1 & 3,3 & 4,3 \\
\hline \multicolumn{6}{|c|}{ Productos químicos } \\
\hline $0,03-0,6$ & 58,8 & 0,0 & 35,3 & 0,0 & 5,9 \\
\hline $0,6-3$ & 64,3 & 3,6 & 21,4 & 7,1 & 3,6 \\
\hline 3 a 15 & 60,0 & 6,7 & 30,0 & 0,0 & 3,3 \\
\hline Más de 15 & 69,3 & 4,0 & 22,7 & 1,3 & 2,7 \\
\hline Total & 67,7 & 4,3 & 23,7 & 1,4 & 2,8 \\
\hline \multicolumn{6}{|c|}{ Bienes de equipo } \\
\hline $0,03-0,6$ & 58,8 & 1,9 & 17,6 & 15,7 & 5,9 \\
\hline $0,6-3$ & 61,4 & 3,5 & 17,5 & 10,5 & 7,0 \\
\hline 3 a 15 & 53,7 & 7,3 & 24,4 & 9,8 & 4,9 \\
\hline Más de 15 & 60,3 & 11,5 & 21,8 & 3,8 & 2,6 \\
\hline Total & 59,1 & 10,0 & 21,9 & 5,6 & 3,4 \\
\hline \multicolumn{6}{|c|}{ Sector del automóvil } \\
\hline $0,03-0,6$ & 60,1 & 0,0 & 39,9 & 0,0 & 0,0 \\
\hline $0,6-3$ & 100,0 & 0,0 & 0,0 & 0,0 & 0,0 \\
\hline 3 a 15 & 40,0 & 0,0 & 40,0 & 20,0 & 0,0 \\
\hline Más de 15 & 80,0 & 16,0 & 4,0 & 0,0 & 0,0 \\
\hline Total & 78,0 & 15,0 & 6,0 & 1,1 & 0,0 \\
\hline \multicolumn{6}{|c|}{ Bienes de consumo duradero } \\
\hline $0,03-0,6$ & 66,6 & 0,0 & 16,7 & 16,7 & 0,0 \\
\hline $0,6-3$ & 60,0 & 0,0 & 40,0 & 0,0 & 0,0 \\
\hline 3 a 15 & 60,0 & 0,0 & 40,0 & 0,0 & 0,0 \\
\hline Más de 15 & 57,1 & 14,3 & 28,6 & 0,0 & 0,0 \\
\hline Total & 58,2 & 9,5 & 32,0 & 0,3 & 0,0 \\
\hline \multicolumn{6}{|c|}{ Manufacturas de consumo } \\
\hline $0,03-0,6$ & 60,7 & 0,0 & 35,7 & 0,0 & 3,6 \\
\hline $0,6-3$ & 53,6 & 7,1 & 28,6 & 7,1 & 3,6 \\
\hline 3 a 15 & 59,3 & 0,0 & 22,2 & 14,8 & 3,7 \\
\hline Más de 15 & 72,4 & 6,9 & 20,7 & 0,0 & 0,0 \\
\hline Total & 67,6 & 5,2 & 21,9 & 4,0 & 1,2 \\
\hline Total & 64,8 & 7,7 & 22,7 & 2,7 & 2,2 \\
\hline
\end{tabular}


superiores al $90 \%$ ) en todos los casos, aunque con diferencias significativas dentro de cada sector, por intervalos de exportación.

En lo que se refiere a la razón principal por la que las empresas deciden no utilizar los servicios de ICEX, a pesar de que los conozcan, se observa también una elevada diversidad intrasectorial cuando se analizan las respuestas por intervalo de exportación. Aunque en todos los casos la causa aducida por un mayor porcentaje de empresas es no necesitar estos servicios, seguida de la falta de adaptación de los instrumentos, las frecuencias de estas y otras respuestas varían en función del intervalo de exportación, dentro de cada sector.

La importancia que las empresas atribuyen a otras posibles razones, como el coste, los requisitos administrativos o la existencia de organismos que ofrecen mejor servicio es también heterogénea ${ }^{3}$. A modo de ejemplo, dentro del sector del automóvil, un $16 \%$ de las empresas con grandes volúmenes de exportación (por encima de 15 millones anuales) apuntan como factor relevante a la existencia de organismos que ofrecen mejores servicios. En los intervalos inferiores de exportación este porcentaje se reduce a cero. Similarmente, un $16,7 \%$ de las empresas exportadoras de bienes de equipo situadas en el intervalo inferior de exportaciones (de 30.000 a 600.000 euros por año) considera que los requerimientos excesivos son la principal razón por la que no han utilizado los

3 Los servicios de apoyo a la internacionalización pueden ser prestados, básicamente, por cuatro tipo de actores: 1) ICEX; 2) los organismos de promoción de las CCAA — gran parte de cuya actividad es canalizada a través de ICEX, como la participación en ferias-; 3) cámaras de comercio, -igualmente, gran parte de los servicios de internacionalización son misiones directas o inversas, que se canalizan a través de los servicios prestados por la Red de Oficinas Económicas y Comerciales, esto es, servicios ICEX-; 4) asociaciones sectoriales, gran parte de cuyos servicios se incluyen en los llamados planes sectoriales y, en muchos casos, son objeto de ayuda de ICEX. En resumen, como ya se ha señalado, a menudo otras entidades de apoyo a la internacionalización prestan servicios canalizados o apoyados de forma indirecta o directa por los programas o estructura de ICEX, perdiendo este visibilidad de cara a la empresa. servicios de ICEX. Este porcentaje es nulo en el resto de los tramos de exportación.

En definitiva, los análisis más desagregados apuntan a una diversidad de situaciones de conocimiento, uso y satisfacción con los servicios de ICEX relacionadas, a su vez, con un conjunto también heterogéneo de razones que determinan que algunas empresas que conocen ICEX no hagan uso de sus servicios. Estos resultados sugieren que la cobertura y eficacia de los servicios de ICEX puede variar en función de las características de las empresas a las que se orientan dichos servicios.

\section{Resultados para el seguro de crédito a la exportación de CESCE}

Los resultados obtenidos (Cuadro 5) revelan que el $13,8 \%$ de las empresas españolas que exportan regularmente valora positivamente la incidencia que ha tenido el seguro de crédito a la exportación ofrecido por CESCE, desde 2017 hasta finales de 2019, en su proceso de internacionalización. Este resultado refleja el porcentaje que representan las empresas que conocen, han utilizado y han quedado satisfechas con el seguro de crédito a la exportación ofrecido por CESCE sobre el total de empresas que forman parte del ámbito de investigación.

Al valorar este resultado es preciso tener en cuenta que se está preguntando a las empresas por un producto específico, el seguro de crédito a la exportación. Este producto incluiría tanto operaciones realizadas por cuenta del Estado, que suelen centrarse en la exportación de bienes de equipo, como operaciones que CESCE realiza por cuenta propia y que cubren un abanico sectorial más amplio.

En todo caso, es razonable que, como se observa en los resultados, los grados de $\triangleright$ 
Subdirección General de Estudios y Evaluación de Instrumentos de Política Comercial

CUADRO 5

PERCEPCIÓN DE LAS EMPRESAS EXPORTADORAS SOBRE LA COBERTURA Y EFICACIA DE CESCE

\begin{tabular}{|c|c|c|c|c|}
\hline \multicolumn{5}{|c|}{ Porcentaje de empresas del sector } \\
\hline Sectores & Conocimiento & Utilización & Satisfacción & +/Total \\
\hline Alimentación, bebidas y tabaco & 72,7 & 22,4 & 95,6 & 15,6 \\
\hline Productos energéticos & 47,3 & 13,3 & 100,0 & 6,3 \\
\hline Materias primas & 62,8 & 31,0 & 99,1 & 19,3 \\
\hline Semimanufacturas no químicas & 77,1 & 31,4 & 93,3 & 22,5 \\
\hline Productos químicos & 64,7 & 28,6 & 86,2 & 15,9 \\
\hline Bienes de equipo & 69,9 & 23,0 & 97,7 & 15,7 \\
\hline Sector del automóvil & 52,1 & 17,4 & 67,7 & 6,1 \\
\hline Bienes de consumo duradero & 56,7 & 42,8 & 95,7 & 23,2 \\
\hline Manufacturas de consumo & 60,2 & 24,3 & 89,4 & 13,1 \\
\hline Total & 64,1 & 23,9 & 90,4 & 13,8 \\
\hline \multicolumn{5}{|c|}{ Porcentaje de empresas del intervalo de exportación } \\
\hline Millones de $€$ & Conocimiento & Utilización & Satisfacción & +/Total \\
\hline $0,03-0,6$ & 57,9 & 20,4 & 91,0 & 10,8 \\
\hline $0,6-3$ & 64,1 & 24,0 & 88,0 & 13,5 \\
\hline 3 a 15 & 64,1 & 24,1 & 96,4 & 14,9 \\
\hline Más de 15 & 64,1 & 23,9 & 89,4 & 13,7 \\
\hline Total & 64,1 & 23,9 & 90,4 & 13,8 \\
\hline
\end{tabular}

conocimiento y utilización de este servicio sean inferiores a los declarados para la amplia gama de ayudas, programas y servicios que ofrece ICEX.

Por sectores, aquellos en los que hay un mayor porcentaje de empresas regulares que se han beneficiado del seguro de crédito a la exportación de CESCE son los bienes de consumo duradero $(23,2 \%)$, las semimanufacturas no químicas $(22,5 \%)$, las materias primas (19,3\%), los productos químicos $(15,9 \%)$ y los bienes de equipo (15,7\%). Aquellos sectores en los que hay un menor porcentaje de empresas que se han beneficiado del seguro de crédito a la exportación de CESCE son los productos energéticos $(6,3 \%)$ y el sector del automóvil $(6,1 \%)$.

Por intervalos de valor de exportación, destaca, con un $14,9 \%$, el porcentaje de empresas que perciben un resultado positivo de CESCE en el intervalo medio alto (de 3 a 15 millones de euros).

El grado de satisfacción de las empresas regulares que conocen y utilizan el seguro de crédito a la exportación de CESCE es elevado en la práctica totalidad de los sectores e intervalos de exportación. Globalmente, el $90,4 \%$ de las empresas usuarias expresan satisfacción con el resultado. La disparidad más notable se da en el sector del automóvil, donde el indicador de satisfacción es del $67,7 \%$. En sentido positivo, destaca el $100 \%$ de satisfacción entre las empresas energéticas. Por intervalos de exportación, los usuarios con mayor grado de satisfacción $(96,4 \%)$ se sitúan en el tramo de 3 a 15 millones de euros.

Al igual que en el caso de los servicios de ICEX, el análisis más desagregado, cruzando las dimensiones sectorial y de intervalo de exportación, refleja una elevada heterogeneidad (Cuadro 6). 
CUADRO 6

COBERTURA Y EFICACIA DE CESCE. DESGLOSE POR SECTOR E INTERVALOS DE EXPORTACIÓN

Porcentaje de empresas del sector e intervalo de exportación (millones de $€$ )

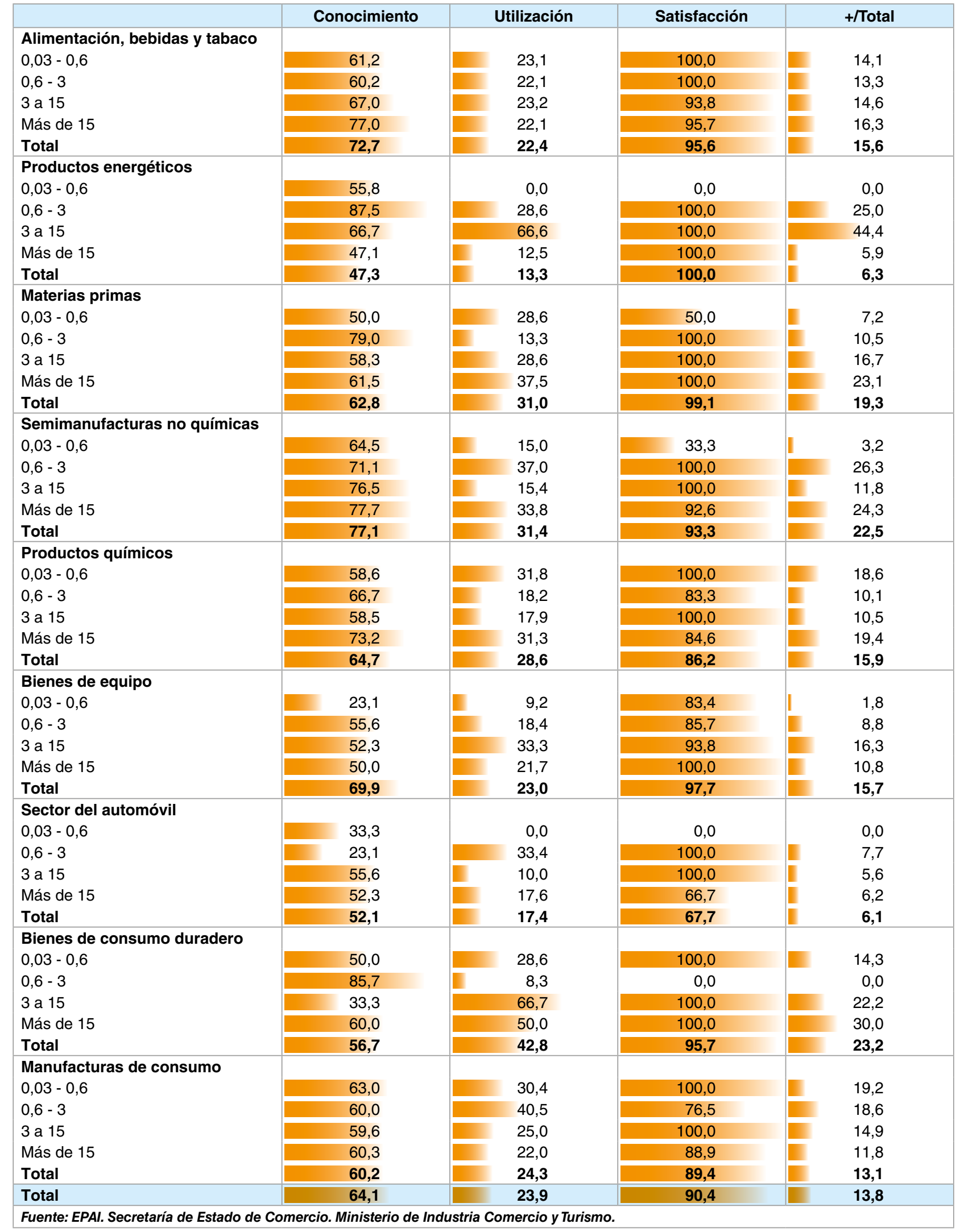


En este caso, no se observa una asociación clara, dentro de cada sector, entre un mayor nivel de exportación de las empresas y un mejor conocimiento del seguro de crédito a la exportación de CESCE. En algunos sectores (por ejemplo, alimentación, bebidas y tabaco o manufacturas no químicas) son las empresas situadas en los tramos más elevados de exportación las que, con mayor frecuencia, declaran conocer este producto de CESCE. En otros sectores (por ejemplo, productos energéticos o bienes de consumo duradero), los porcentajes de conocimiento más elevados se dan en el tramo de 600.000 a 3 millones de euros de exportación.

En lo que se refiere a la frecuencia de uso entre las empresas de cada sector que declaran conocer el seguro de crédito a la exportación de CESCE y al grado de satisfacción entre los usuarios, se observa también una cierta diversidad de respuestas para los distintos intervalos de exportación. Sí es posible determinar que algunos resultados observados a nivel sectorial, como el grado de satisfacción comparativamente reducido del automóvil, se concentran en un determinado intervalo de exportación.

En definitiva, los análisis más desagregados sugieren que la heterogeneidad empresarial puede ser también un factor determinante en la percepción, por parte de las empresas, de la utilidad del seguro de crédito a la exportación.

\section{Conclusiones}

El ejercicio piloto realizado a través de la EPAI permite obtener una visión muy general de cómo perciben las empresas que exportan regularmente los servicios ofrecidos por dos entidades que contribuyen al apoyo a la internacionalización, ICEX y CESCE. Este análisis nos permite alcanzar algunas conclusiones preliminares, que deberán ser confirmadas o matizadas por las evaluaciones más en profundidad que hacen estas instituciones de sus principales instrumentos. Además, la EPAI limita su alcance a las empresas exportadoras regulares, quedando fuera un número importante de empresas cuyas características y necesidades pueden ser distintas.

En ambos casos, el grado de satisfacción de los usuarios con los servicios utilizados puede considerarse muy elevado, de forma que cabe concluir que ICEX y CESCE prestan servicios de elevada calidad a sus usuarios. No obstante, el objetivo de este ejercicio es identificar aquellos ámbitos en los que pueda haber margen para seguir mejorando, en particular, para potenciar una mayor cobertura y eficacia de los apoyos a la internacionalización.

En este sentido, parece conveniente continuar reforzando la visibilidad, tanto de ICEX como de CESCE, al menos entre las empresas que exportan regularmente, dado que el grado de conocimiento declarado es elevado pero mejorable. En particular, parece oportuno seguir poniendo el énfasis en la difusión de estos instrumentos entre los grupos de empresas con cifras de exportación modestas, donde, probablemente, abundan las pymes.

Adicionalmente, sería recomendable una mayor adaptación de los apoyos, programas y servicios ofrecidos a las necesidades de las empresas. En este sentido, los resultados de la EPAI relativos a las razones que aducen las empresas para no haber utilizado estos servicios sugieren que una mayor adaptación podría fomentar su uso, al menos para el caso de ICEX. Por otro lado, tanto en el caso de ICEX como de CESCE, la heterogeneidad de resultados por sectores e intervalos de exportación apunta en el mismo sentido. 
Para avanzar en estas líneas es necesario mejorar el conocimiento de las características de las empresas internacionalizadas y con potencial para la internacionalización, así como continuar desarrollando instrumentos que permitan conocer las opiniones de estas empresas sobre los servicios de apoyo a la internacionalización y medir sus efectos.

Es importante tener en cuenta que la EPAI se ha realizado en el marco del proceso de evaluación del Plan de Acción para la Internacionalización $2017 / 18$, que es el periodo que se pretende evaluar para obtener recomendaciones a aplicar en futuros planes. El Plan de Acción 2019/2020, en aplicación del eje 1 de la Estrategia Española de Internacionalización, ya destaca estas cuestiones, de forma que ya están siendo tenidas en cuenta por las entidades correspondientes.

Las instituciones responsables de los servicios de apoyo a la internacionalización vienen trabajando intensamente en esta línea. En este sentido están consolidando la implantación de herramientas de gestión, análisis y medición, como el Customers Relationship Managment $(\mathrm{CMR})$, las herramientas de analítica y big data, o de medición de la satisfacción del cliente a través del Net Promoter Score (NPS). La aplicación de este tipo de herramientas facilita una medición continua de la actividad y eficiencia de los organismos, un buen conocimiento del número y tipo de clientes que es usuario de cada servicio, de su satisfacción con el servicio utilizado, e incluso, a través de un adecuado seguimiento de las empresas y del impacto de estos servicios en su proceso de internacionalización. En definitiva, estas herramientas potencian un mejor conocimiento del cliente y una capacidad de análisis más potente que facilita la adecuación de cada servicio concreto a las necesidades de los clientes.

La EPAI es un pequeño paso en esta dirección. Al proporcionar una visión global sobre las percepciones de las empresas exportadoras complementa las actuaciones que están ejecutando las instituciones responsables de los distintos instrumentos de apoyo a la internacionalización para conocer mejor a sus clientes y responder a sus necesidades.

\section{Bibliografía}

Ministerio de Economía, Industria y Competitividad (2017). La Estrategia de Internacionalización de la Economía Española 2017-2027. http://www. mineco.gob.es/stfls/mineco/comercio/pdf/ 170913_Estrategia_Internacionalizacion_2017. pdf

Subdirección General de Análisis y Estrategia de Internacionalización (2017). La estrategia de internacionalización de la economía española 20172027. Boletín Económico de ICE (3093). https:// doi.org/10.32796/bice.2017.3093.5674

Subdirección General de Estrategia de Internacionalización (2019). El Plan de Acción para la Internacionalización de la Economía Española 2019-2020. Boletín Económico de ICE (3111). https://doi.org/10.32796/bice.2019.3111.6827 


\section{ANEXO \\ CUESTIONARIO DE LA ENCUESTA PILOTO DE APOYO A LA INTERNACIONALIZACIÓN}

N1. ¿Conocen en su empresa la entidad ICEX España Exportación e Inversiones?

\begin{tabular}{|c|c|}
\hline Sí & Ir a la pregunta 2. \\
\hline No & Ir la pregunta 5. \\
\hline
\end{tabular}

N2. ¿Ha utilizado su empresa algún tipo de ayuda, programa o servicio de ICEX España Exportación e Inversiones desde el 1 de enero de 2017 en adelante?

\begin{tabular}{|c|c|}
\hline Sí & Ir a la pregunta 3. \\
\hline No & Ir la pregunta 4. \\
\hline
\end{tabular}

N3. ¿Quedó su empresa satisfecha con la ayuda, programa o servicio prestado por ICEX?

\begin{tabular}{|c|c|}
\hline Sí & Ir a la pregunta 5. \\
\hline No & Ir la pregunta 5. \\
\hline
\end{tabular}

N4. Señale el principal motivo por el que su empresa no ha utilizado ningún apoyo o servicio de ICEX España Exportación e Inversiones.

\begin{tabular}{|l|l|}
\hline \multicolumn{1}{|c|}{ Instrumentos ICEX } & Marca \\
\hline No necesito ninguno de sus instrumentos & \\
\hline Estos instrumentos están mejor cubiertos por otro organismo & \\
\hline Los instrumentos no se adaptaban a las necesidades de mi empresa & \\
\hline Los requerimientos de acceso son excesivos & \\
\hline Los servicios ofrecidos eran demasiado caros & \\
\hline
\end{tabular}

N5. ¿Conocen en su empresa el seguro de crédito a la exportación de CESCE?

\begin{tabular}{|c|c|}
\hline Sí & Ir a la pregunta 6. \\
\hline No & FIN \\
\hline
\end{tabular}

N6. ¿Ha utilizado algún tipo de cobertura de CESCE asociada a la exportación desde el 1 de enero de 2017 en adelante?

\begin{tabular}{|c|c|}
\hline Sí & Ir a la pregunta 7. \\
\hline No & FIN \\
\hline
\end{tabular}

N7. ¿Quedó su empresa satisfecha con el servicio prestado por CESCE?

\begin{tabular}{|c|c|}
\hline Sí & FIN \\
\hline No & FIN \\
\hline
\end{tabular}

